\title{
Spin-polarized current amplification and spin injection in magnetic bipolar transistors
}

\author{
Jaroslav Fabian \\ Institute for Theoretical Physics, Karl-Franzens University, Universitätsplatz, 5, 8010 Graz, Austria \\ Igor Žutić* \\ Condensed Matter Theory Center, Department of Physics, University of Maryland at College Park, \\ College Park, Maryland 20742-4111, USA
}

(Received 27 November 2002; revised manuscript received 19 November 2003; published 12 March 2004)

\begin{abstract}
The magnetic bipolar transistor (MBT) is a bipolar junction transistor with an equilibrium and nonequilibrium spin (magnetization) in the emitter, base, or collector. The low-injection theory of spin-polarized transport through MBT's and of a more general case of an array of magnetic $p$ - $n$ junctions is developed and illustrated on several important cases. Two main physical phenomena are discussed: electrical spin injection and spin control of current amplification (magnetoamplification). It is shown that a source spin can be injected from the emitter to the collector. If the base of a MBT has an equilibrium magnetization, the spin can be injected from the base to the collector by intrinsic spin injection. The resulting spin accumulation in the collector is proportional to $\exp \left(q V_{b e} / k_{B} T\right)$, where $q$ is the proton charge, $V_{b e}$ is the bias in the emitter-base junction, and $k_{B} T$ is the thermal energy. To control the electrical current through MBT's both the equilibrium and the nonequilibrium spin can be employed. The equilibrium spin controls the magnitude of the equilibrium electron and hole densities, thereby controlling the currents. Increasing the equilibrium spin polarization of the base (emitter) increases (decreases) the current amplification. If there is a nonequilibrium spin in the emitter, and the base or the emitter has an equilibrium spin, a spin-valve effect can lead to a giant magnetoamplification effect, where the current amplifications for the parallel and antiparallel orientations of the equilibrium and nonequilibrium spins differ significantly. The theory is elucidated using qualitative analyses and is illustrated on a MBT example with generic materials parameters.
\end{abstract}

DOI: 10.1103/PhysRevB.69.115314

PACS number(s): 72.25.Dc, 72.25.Mk

\section{INTRODUCTION}

Integrating charge and spin properties of semiconductors is the central goal of semiconductor spintronics ${ }^{1}$ whose prospect has been fueled by the experimental demonstration of electrical spin injection into semiconductors, ${ }^{2-6}$ as well as by the discovery of III-V ferromagnetic semiconductors ${ }^{7,8}$ (Eubased ferromagnetic semiconductors have even been used earlier as effective spin filters ${ }^{9,10}$ ) and observations of relatively long spin relaxation times. ${ }^{11,12}$ Many important advances have already been made toward an efficient spin control of electrical current in semiconductors and, vice versa, control of magnetism by electrical means. Recent examples include a control of ferromagnetism by incident light ${ }^{13,14}$ or by gate voltage, ${ }^{15,16}$ spin injection induced magnetoresistance in nonmagnetic semiconductors, ${ }^{17}$ or the spin-galvanic effect. $^{18}$

Transistors are naturally suited for spin control of electrical currents since the three regions, emitter, base, and collector, can serve as a spin injector, transport medium, and spin detector, respectively. There has been remarkable experimental progress by employing hybrid ferromagnetic metal and semiconductor structures as the hot-electron transistors; ${ }^{19}$ the magnetoresistance of such transistors can be as large as $3400 \%$ (Ref. 20) and they can be used as effective spin injectors. $^{21}$ The theoretical proposals for spin transistors focus largely on the field-effect systems. ${ }^{22-24}$ In this paper we analyze magnetic bipolar transistors (MBT's) which are conventional (spin-unpolarized) bipolar junction transistors ${ }^{25}$ with added spin. ${ }^{26}$ MBT's were first proposed in Ref. 27 (see also Ref. 28) where we analyzed spin injection and current amplification of npn MBT's with a source spin. ${ }^{29}$ Special cases of MBT's without a source spin were recently studied by Flatte et al. ${ }^{30}$ who calculated the spin current polarization in a magnetic-base npn MBT, and by Lebedeva and Kuivalainen, ${ }^{31}$ who calculated the current amplification in a magnetic emitter pnp MBT. Of spin transistors, the closest one to MBT is the so-called SPICE (spin-polarized injection current emitter), ${ }^{32}$ which employs ferromagnetic metals in the emitter and base-collector regions. One of the principle drives for proposing all-semiconductor spin transistors is the possibility of controlling current amplification by spin.

MBT's integrate ferro(magnetic) and nonmagnetic semiconductors in the usual bipolar junction transistor geometry. ${ }^{25,33}$ Material and electrical properties of hybrid ferromagnet/semiconductor heterostructures are currently an active area of research. ${ }^{34}$ The potential of ferromagnetic semiconductors for bipolar devices has been shown already in Ref. 35 where a ferromagnetic diode was presented. ${ }^{36}$ More recently (Ga,Mn)As/GaAs $p-n$ heterojunctions have been fabricated ${ }^{37}$ and electrical spin injection through magnetic bipolar tunnel junctions has been demonstrated ${ }^{38,39}$ showing up to $\approx 80 \%$ injected electron-density spin polarization at $4.6 \mathrm{~K} .{ }^{40}$ Finally, in Ref. 41 a CoMn doped $p$-Ge and an $n$-Ge were put together to form a ferromagnetic $p$ - $n$ heterojunction which showed magnetization dependent current rectification, with up to $97 \%$ electrical current variations due to the applied magnetic field. Such hybrid junctions can also be used for MBT's, where the requirement is that the magnetic region has a sizable equilibrium spin polarization (say, 
$10 \%)$. This polarization can be provided by the exchange splitting in ferromagnetic semiconductors or by the large Zeeman splitting of dilute magnetic semiconductors. For example, Zeeman splitting can be significantly enhanced by large effective $g$ factors in magnetically doped $(|g| \sim 500$ in $\mathrm{Cd}_{0.95} \mathrm{Mn}_{0.05} \mathrm{Se}$ at low temperatures) or in narrow-band-gap semiconductors $[|g| \approx 50$ in InSb (Ref. 42) even at room temperature]. Another possibility would be to use a ferromagnetic semiconductor slightly above its Curie temperature, a regime also expected to give large $g$ factors. However, before there is an additional progress in fabricating junctions using reported room-temperature ferromagnetic semiconductors [for example, $(\mathrm{Zn}, \mathrm{Cr}) \mathrm{Te}$ (Ref. 43)], the demonstration of the operation of MBT's will likely be limited to temperatures below $\sim 150 \mathrm{~K}^{44}$ Room-temperature MBT is certainly a great challenge.

We formulate here a fully analytical theory of spinpolarized transport through MBT's in the small bias (lowinjection) regime, where the injected carrier densities are smaller than the equilibrium ones. The theory uses the generalized Shockley model for the spin-polarized transport through magnetic $p$ - $n$ junctions, ${ }^{45}$ as well as the theory of conventional bipolar junction transistors, as developed by Shockley. ${ }^{25,33}$ Our theory can thus be viewed as a generalized Shockley theory of bipolar transistors. Two different phenomena are studied in detail: electrical spin injection from the emitter to the collector and spin control of the current amplification (also called gain). Electrical spin injection is shown to be effective in the amplification mode of the transistor, the mode where the transistor amplifies current. Spin control can be achieved by modifying both the equilibrium and nonequilibrium spin, since both can modify the electrical current. The control by the equilibrium spin (what we call the magnetoamplification effect) results from the dependence of the equilibrium minority carrier density on the equilibrium spin polarization, while the control by the nonequilibrium spin (what we call the giant magnetoamplification effect) controls the current via the spin-charge coupling of the Silsbee-Johnson type. ${ }^{46,47}$

We first describe the model of MBT's in Sec. II and formulate the analytical theory in Sec. III, leaving the formal aspects of the theory for the Appendix. We then apply the theory to study electrical spin injection through MBT's in Sec. IV, and spin control of the current amplification in Sec. $\mathrm{V}$, where we also discuss the spin current in MBT's.

\section{MODEL}

A conventional, spin-unpolarized bipolar $n p n$ transistor $^{33}$ consists of $n, p$, and $n$ regions connected in series (consult Figs. 1 and 2). Typically the $n$ region with the higher donor doping is called the emitter, the one with the lower doping the collector. The base is the $p$ region (doped with acceptors) sandwiched in between. The most useful mode of operation of the transistor is the so-called amplification (also forward active) mode, where the emitter-base $(b-e)$ junction is forward biased, so that the electrons are easily injected into the base. Together with the opposite flow of holes, they form the emitter current $j_{e}$. The electrons injected into the base dif-

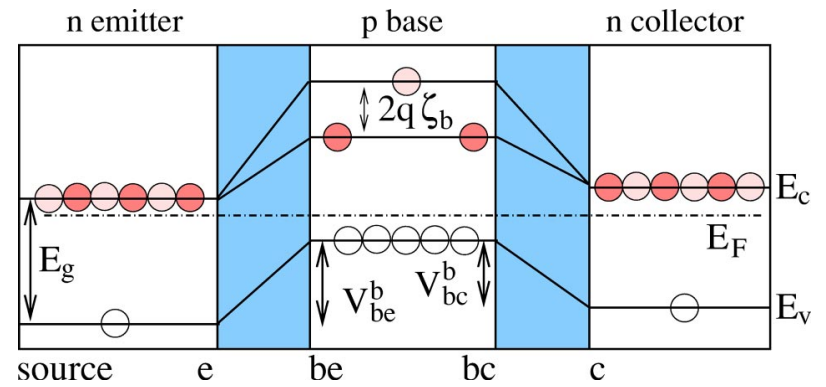

FIG. 1. Scheme of a magnetic npn transistor in equilibrium. The conduction band $E_{c}$ is populated mostly with electrons (filled circles) in the emitter and collector. In this example the base is magnetic with the conduction-band spin split by $2 q \zeta_{b}$. The valence band $E_{v}$ separated by the energy gap $E_{g}$ from the conduction band is populated mostly by holes (empty circles) in the base. The small population of electrons in the base has an equilibrium spin polarization $\alpha_{0 b}=\tanh \left(q \zeta_{b} / k_{B} T\right)$, holes are assumed spin unpolarized. The electron spin is indicated by the dark (spin-up) and light (spindown) shadings. The Fermi level (chemical potential) $E_{F}$ is uniform. Between the bulk regions, built-in potentials $V_{b e}^{b}$ and $V_{b c}^{b}$ are formed defining the depletion layers (shaded) in the base-emitter $(b-e)$ and base-collector $(b-c)$ junctions, respectively. Finally, labels "source," $e, b e, b c$, and $c$ stand for the regions at which they are shown. For example, $b e$ is the region in the base at the boundary with the depletion layer.

fuse towards the collector. The base-collector $(b-c)$ junction is reverse biased. This means that any electron reaching the junction from the base is swept by the electric field in the depletion layer to the collector, forming the electron current (holes's contribution is negligible). The base current is the difference $j_{b}=j_{c}-j_{e}$. This difference comes from two

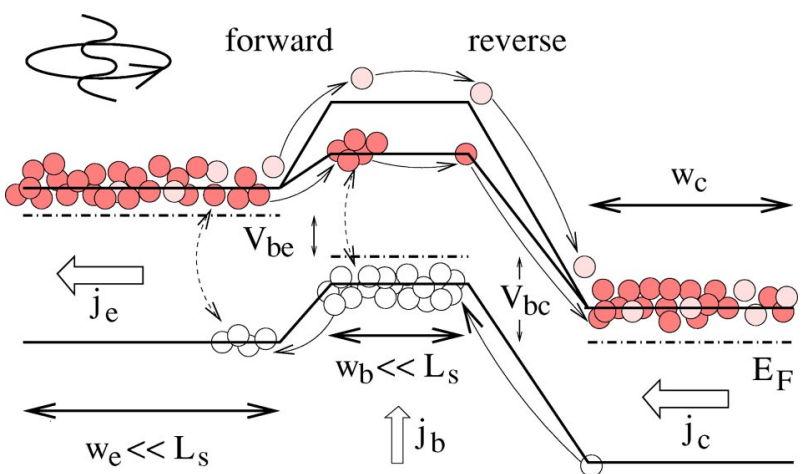

FIG. 2. Scheme of the magnetic npn transistor from Fig. 1 in the amplification mode. The $b-e$ junction is forward biased with $V_{b e}$ $>0$, lowering the barrier and reducing the depletion layer width. The $b-c$ junction is reverse biased with $V_{b c}<0$, raising the barrier and increasing the depletion layer width. The corresponding changes to the Fermi level $E_{F}$ are indicated. The emitter has a spin source, indicated here by the incident circularly polarized light generating nonequilibrium electron spin well within the spin-diffusion length $L_{s}$ from the $b$-e depletion layer. The electron and hole flow gives the emitter $\left(j_{e}\right)$, base $\left(j_{b}\right)$, and collector $\left(j_{c}\right)$ charge currents. The electron-hole recombination is depicted by the dashed lines. Also shown are the effective widths of the emitter $\left(w_{e}\right)$, base $\left(w_{b}\right)$, and collector $\left(w_{c}\right)$. 
sources. First, from the hole current which is present in the emitter but not in the collector. Second, from the electronhole recombination in the base which diminishes the number of electrons that make it to the collector. These two factors form the generally small $j_{b}$. The current amplification $\beta$ is defined as the ratio of the large collector current to the small base current. For practical transistors $\beta$ is of order 100, meaning that small variations in $j_{b}$ (input signal) lead to large variations in $j_{c}$ (output signal). To maximize the gain one needs to (i) minimize the relative contribution of holes in $j_{e}$ or (ii) inhibit the electron-hole recombination in the base. Typically silicon is used to make bipolar transistors, since the indirect gap makes it a poor material for the electron-hole recombination. We will show below that MBT's allow spin control of the gain by realizing (i).

The magnetic bipolar transistor is a bipolar junction transistor with equilibrium spin due to spin-split carrier bands, as well as with a nonequilibrium source spin introduced, for example, by external electrical spin injection or optical orientation. ${ }^{48}$ The equilibrium spin can be a result of the Zeeman splitting in an applied magnetic field or of the exchange splitting due to ferromagnetic semiconductors integrated into the device structure. For our purposes the equilibrium spin splitting should be on the order of thermal energy for the spin-charge coupling discussed below to be significant. If no equilibrium spin is present, this restriction becomes irrelevant, but the spin effects are limited to electrical spin injection.

A MBT in equilibrium is described in Fig. 1. The base is magnetic, with the spin splitting $2 q \zeta_{b}$. In the emitter the majority carriers are electrons whose number is essentially $N_{d e}$, the donor density. Similarly in the collector, where the donor density is $N_{d c}$. Holes are the minority carriers in the two regions. The base is doped with $N_{a b}$ acceptors. Holes (electrons) are the majority (minority) carriers. We assume that only electrons are spin polarized. The inclusion of the hole spin polarization is straightforward and adds no new physics to our considerations. Furthermore, in many important semiconductors (such as GaAs) holes lose their spin orientation very fast ${ }^{49}$ and indeed can be treated as unpolarized. Note that the electron density is $n=n_{\uparrow}+n_{\downarrow}$, the (electron) spin density is $s=n_{\uparrow}-n_{\downarrow}$, and the spin polarization is $\alpha$ $=s / n$. (Spin polarization of the charge current is studied in Sec. V C.)

The equilibrium in MBT's can be disturbed by applying a bias as well as by introducing a nonequilibrium source spin. Figure 2 depicts the nonequilibrium physics and introduces the relevant notation. We assume that the source spin is injected into the emitter within the spin-diffusion length from the $b$-e depletion layer so that enough spin can diffuse to the base. At the $b-e$ depletion layer the electrons feel a spindependent barrier: in Fig. 2 the barrier is smaller (larger) for the spin-up (down) electrons. As in the conventional bipolar transistors there is a significant accumulation of the minority carriers around the forward biased $b-e$ depletion layer, while there are few carriers around the reverse biased $b-c$ layer. The widths $w$ of the bulk regions depend on the applied voltages as well as on the equilibrium spin polarization. ${ }^{45}$

We assume that the electron-hole recombination, occur- ring mostly in the emitter and the base is spin independent, a reasonable approximation for unpolarized holes. We also assume that the spin splitting is uniform in the bulk regions, eliminating magnetic drift (magnetic drift in semiconductors is discussed in Ref. 45). Our other assumptions are those of the standard Shockley theory: ${ }^{25,33}$ Temperature is large enough (say, $T \geqslant 50 \mathrm{~K}$ ) for all the donors and acceptors to be ionized; the carriers obey the nondegenerate Boltzmann statistics; the injected minority carrier densities are much smaller than the equilibrium densities; the electric fields in the bulk regions are small eliminating electrical drift. Furthermore, we neglect the carrier recombination and spin relaxation inside the depletion layers. These effects are important at very low biases and are not relevant for our observations, although may by themselves lead to nice physics. Finally, the contacts with the external electrodes are ohmic, maintaining the carrier (but not necessarily spin) densities in equilibrium.

\section{THEORY}

We generalize the Shockley theory of bipolar transistors to include spin. The theory is valid in the small bias regime and is applicable to any operational mode of the transistor, not only to the amplification regime. Physically, the theory describes electron and hole carrier and spin diffusion in the bulk regions, limited by the electron-hole recombination and spin relaxation. The depletion layers provide only boundary conditions for the diffusion, by connecting the charge and spin currents in the adjacent regions. The most essential assumption is that the spin-resolved chemical potentials remain constant across the depletion layers.

The transistor is viewed as two $p-n$ junctions in series. The minority carrier density in each junction $(b-e$ and $b-c)$ is determined by the bias voltage across each junction. In MBT's the densities are determined also by the spin polarization, which needs to be calculated self-consistently, as is explained below. Within the limits of the theory it is enough to know the minority electron densities $n_{b e}$ and $n_{b c}$ to determine the electron charge currents, and $p_{e}$ and $p_{c}$ to determine the hole charge currents (see Fig. 1 for labeling the regions). We divide the presentation of the theory into two steps. First, we recall the main results of the generalized Shockley theory of magnetic $p-n$ junctions ${ }^{45}$ and second, we use these results to formulate a theory of a series of magnetic $p$ - $n$ junctions and solve it for $n p n$ MBT. The first step is necessary to also understand our qualitative analyses of the transistor operations in the amplification mode. The second step, which is rather technical, is left for the Appendix.

In the following we present selected results of the theory of magnetic $p-n$ junctions. The notation, which is easily adapted for use in MBT's, is summarized in Fig. 3. Both the $p$ and $n$ regions are in general magnetic, biased with voltage $V$. The nonohmic contact (to simulate the conditions at the base of a transistor) at the $p$ region maintains nonequilibrium electron $n_{p}$ and spin $s_{p}$ densities. Similarly, there are nonequilibrium densities $n_{L}$ and $s_{L}$ at the left of the depletion layer. In the $n$ region electrons are the majority carriers whose densities can be considered fixed by the donor density 


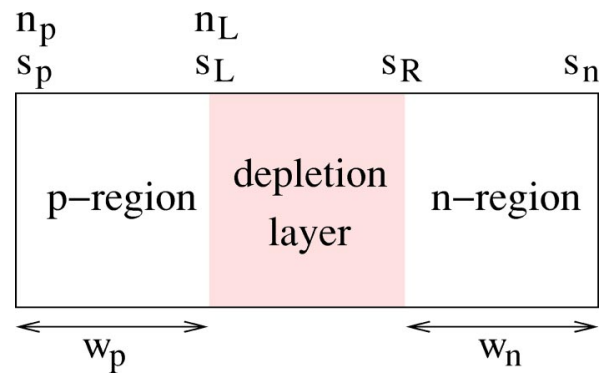

FIG. 3. Magnetic $p$ - $n$ junction. The input densities are the carrier and spin densities at the end of the $p$ region, $n_{p}$ and $s_{p}$, as well as at the end of the $n$ region, $s_{n}$. The densities to be calculated are at the edge of the depletion layer: $s_{L}$ in the $p$ and $s_{R}$ in the $n$ region. Also indicated are the effective widths of the two bulk regions.

$N_{d}$. However, the spin density can vary, being $s_{R}$ at the right of the depletion layer and $s_{n}$ at the contact with the external ohmic electrode. We use subscript 0 to denote equilibrium quantities. The equilibrium minority densities are $n_{0 p}$ (electrons in $p$ ), $p_{0 n}$ (holes in $n$ ), and $s_{0 p}$ (electron spin in $p$ ). These densities are uniform across the corresponding regions. The equilibrium density spin polarizations in the $n$ and $p$ regions are $\alpha_{0 n}$ and $\alpha_{0 p}$, respectively. These are also uniform. We denote the excess (injected) quantities by $\delta$. For example, $\delta s_{L}=s_{L}-s_{0 p}$. We next denote by $L_{n p}$ the electron diffusion length in $p$, and by $L_{s n}$ and $L_{s p}$ the electron spindiffusion length in $n$ and $p$, respectively. Finally, $D_{n p}\left(D_{n n}\right)$ stand for the electron diffusion coefficients in $n(p)$. Similarly for holes.

The spin injection efficiency in magnetic $p-n$ junctions is measured by $\alpha_{R}=\delta s_{R} / N_{d}$, where ${ }^{45}$

$$
\begin{aligned}
\delta s_{R}= & \gamma_{0} \delta s_{n}+\gamma_{1}\left(\delta s_{p}-\alpha_{0 p} \delta n_{p}\right)+\gamma_{2} \alpha_{0 L} \delta n_{p} \\
& -\gamma_{2} \cosh \left(w_{p} / L_{n p}\right) s_{0 L}\left(e^{q V / k_{B} T}-1\right)
\end{aligned}
$$

the transport/geometry $\gamma$ factors are

$$
\begin{gathered}
\gamma_{0}=\frac{1}{\cosh \left(w_{n} / L_{s n}\right)}, \\
\gamma_{1}=\left(\frac{D_{n p} L_{s n}}{D_{n n} L_{s p}}\right) \frac{\tanh \left(w_{n} / L_{s n}\right)}{\sinh \left(w_{p} / L_{s p}\right)}, \\
\gamma_{2}=\left(\frac{D_{n p} L_{s n}}{D_{n n} L_{n p}}\right) \frac{\tanh \left(w_{n} / L_{s n}\right)}{\sinh \left(w_{p} / L_{n p}\right)} .
\end{gathered}
$$

Equation (1) is accurate up to the terms of the relative order of $n_{0} \exp \left(q V / k_{B} T\right) / N_{d}$. While such terms can be safely neglected when dealing with the spin and carrier densities, they must be included when calculating the spin current in the $n$ region, where a difference between two small spin densities of the same order needs to be evaluated. (These terms are not presented in Ref. 45.) The exact formula for the injected spin density $\delta s_{R}$ can be cast in the form of Eq. (1), but with the coefficients $\gamma$ divided by the factor $1+\nu$ :

$$
\gamma \rightarrow \gamma /(1+\nu),
$$

where

$$
\begin{gathered}
\nu=\frac{n_{0 p} e^{q V / k_{B} T}}{N_{d}}\left[\gamma_{1} \cosh \left(w_{p} / L_{s p}\right) \frac{1-\alpha_{0 p}^{2}}{1-\alpha_{0 n}^{2}}\right. \\
\left.+\gamma_{3} \alpha_{0 p} \frac{\alpha_{0 p}-\alpha_{0 n}}{1-\alpha_{0 n}^{2}}\right] .
\end{gathered}
$$

Typically $\nu$ is a number smaller than 0.1 , so the corrections to the spin injected density are upmost $10 \%$. Knowing $\delta \alpha_{R}$ we can calculate the injected minority densities $\delta n_{L}$ and $\delta s_{L}$ :

$$
\begin{gathered}
\delta n_{L}=n_{0 p}\left[e^{q V / k_{B} T}\left(1+\delta \alpha_{R} \frac{\alpha_{0 p}-\alpha_{0 n}}{1-\alpha_{0 n}^{2}}\right)-1\right], \\
\delta s_{L}=s_{0 p}\left[e^{q V / k_{B} T}\left(1+\frac{\delta \alpha_{R}}{\alpha_{0 p}} \frac{1-\alpha_{0 p} \alpha_{0 n}}{1-\alpha_{0 n}^{2}}\right)-1\right] .
\end{gathered}
$$

The following relation connects the spin polarization across the depletion layer:

$$
\alpha_{L}=\frac{\alpha_{0 p}\left(1-\alpha_{0 n}^{2}\right)+\delta \alpha_{R}\left(1-\alpha_{0 p} \alpha_{0 n}\right)}{1-\alpha_{0 n}^{2}+\delta \alpha_{R}\left(\alpha_{0 p}-\alpha_{0 n}\right)} .
$$

Equations (1) and (9)-(11) will be referred to as the magnetic $p$ - $n$ junction equations.

In the second step we wish to generalize the magnetic $p-n$ junction equations to the case of several magnetic $p-n$ junctions in series. Such a generalization is straightforward in the unpolarized case, where each junction acts independently from the others, since the minority carrier densities are fixed only by $V$. The inclusion of spin complicates the matter in the following sense. In a single junction $\delta n_{p}, \delta s_{p}$, and $\delta s_{n}$ are the known boundary conditions, fully determining $\delta s_{R}$ and $\delta s_{L}$. Suppose we now connect two junctions as in the $n p n$ MBT in Fig. 1. Take the $b-c$ junction to be the one in Fig. 3. Densities $n_{p}$ and $s_{p}$ become $n_{b e}$ and $s_{b e}$, themselves unknown, so that $\delta s_{R}$ (now $\delta s_{c}$ ) is undetermined. On the other hand, considering $b-e$ to be the junction in Fig. $3, s_{R}$ becomes $s_{e}\left(s_{n}\right.$ becomes the spin source density), and $n_{L}$ and $s_{L}$ become $n_{b e}$ and $s_{b e}$. These three densities are determined also from $n_{p}$ and $s_{p}$, which are now denoted as $n_{b c}$ and $s_{b c}$. This loop shows the need to obtain the densities inside the transistor (or a more general junction device) selfconsistently. Charge and spin are coupled both across the depletion layers [through Eqs. (9) and (10)]—intrajunction coupling - as well as across the bulk regions between two depletion layers-interjunction coupling. This theory is formally developed in the Appendix.

In the following we consider specific applications of the theory. Since we will deal mostly with the amplification mode where the excess densities in the $b-c$ junction are negligible, we can get useful insights even without the selfconsistent solutions, using only the results presented in this section. We refer to this as qualitative analysis. However, we support each case using a numerical example of a generic MBT, calculated with the full theory presented in the Appendix. The numerical model is a "silicon"-based MBT with the 
following room-temperature parameters. Since the main features of the full theory are captured by the qualitative formulas, one can easily check the properties of MBT's with different parameters. The parameters given below, while generic, are for illustration only. Unless specified otherwise, the nominal widths of the emitter, base, and collector are $2 \mu \mathrm{m}, 1 \mu \mathrm{m}$, and $2 \mu \mathrm{m}$, respectively. The donor doping densities of the emitter and collector are $N_{d e}=10^{17} \mathrm{~cm}^{-3}$ and $N_{d c}=10^{15} \mathrm{~cm}^{-3}$, while the acceptor density in the base is $N_{a b}=10^{16} \mathrm{~cm}^{-3}$. The intrinsic carrier density at room temperature is $n_{i}=10^{10} \mathrm{~cm}^{-3}$. The carrier and spinrelaxation times are taken to be $100 \mathrm{~ns}$ and $10 \mathrm{~ns}$, and the electron (hole) diffusion coefficients $D_{n}=100\left(D_{p}\right.$ =10) $\mathrm{cm}^{2} \mathrm{~s}^{-1}$, all uniform throughout the sample. The carrier and spin-diffusion lengths are $L_{n p}=\left(D_{n p} \tau\right)^{0.5} \approx 30 \mu \mathrm{m}$, $L_{p n}=\left(D_{p n} \tau\right)^{0.5} \approx 10 \mu \mathrm{m}, \quad L_{s n}=\left(D_{n n} T_{1}\right)^{0.5} \approx 10 \mu \mathrm{m}, \quad$ and $L_{s p} \approx L_{s n}$. The dielectric constant is 12 . We assume a spin "ohmic" contact $(\delta s=0)$ at the end of the collector, while at the end of the emitter an external spin injection gives $\delta s^{\text {source }} \neq 0$ in general.

\section{ELECTRICAL SPIN INJECTION}

Electrical spin injection through MBT will be studied in two cases: spin injection of the source spin from the emitter to the collector and spin injection into the collector from the equilibrium spin in the base. The spin injection efficiency in both cases is proportional to the Boltzmann factor $\exp \left(q V_{b e} / k_{B} T\right)$, but the physics behind them is rather disparate. Unless specified otherwise, we work in the amplification mode, where $V_{b e} \gg k_{B} T$ (forward bias) and $V_{b c} \leqslant 0$ (reverse bias), and in the thin base limit, where $w_{b} \ll L_{n b}, L_{s b}$.

\section{A. Source spin}

Suppose a source spin density $\delta s^{\text {source }}$ of polarization $\delta \alpha^{\text {source }}=\delta s^{\text {source }} / N_{d e}$ is injected into the emitter. What is the spin response in the collector? Consider first a nonmagnetic case ( $\alpha_{0}=0$ everywhere). The spin injection involves three steps.

(i) The source spin diffusion towards $b$-e. At the depletion layer the nonequilibrium spin is $\delta s_{e}=\gamma_{0, b e} \delta s^{\text {source }}$. The spin polarization is $\delta \alpha_{e}=\delta s_{e} / N_{d e}$. Note that be in $\gamma_{i, b e}$ $\left(\gamma_{i, b c}\right)$ means that $\gamma_{i}$ given in Eqs. (3)-(5) are evaluated for the $b-e(b-c) p-n$ junction. Since we assume that $L_{s e} \gtrsim w_{e}$, it follows that $\delta \alpha_{e} \approx \delta \alpha^{\text {source }}$.

(ii) Transfer of the spin into the base. From Eq. (11) it follows that $\alpha_{e}=\alpha_{b e}$, showing the efficiency of the spin injection by the majority electrons. The corresponding spin density is $\delta s_{b e}=\delta \alpha_{b e} n_{0 b} \exp \left(q V_{b e} / k_{B} T\right)$, as follows from Eq. (10) for the forward bias case.

(iii) Spin injection into the collector. Equation (1) implies that $\delta s_{c}=\gamma_{1, b c} s_{b e}$ and so the nonequilibrium spin polarization in the collector is $\alpha_{c}=\gamma_{1, b c} s_{b e} / N_{d c}$, a result of the minority electrons spin pumping: ${ }^{50}$ spin in the base diffuses towards the reverse biased depletion layer where it is swept by the built-in field to the collector. Here the spin density accumulates as it is bottlenecked by spin diffusion and spin relaxation.

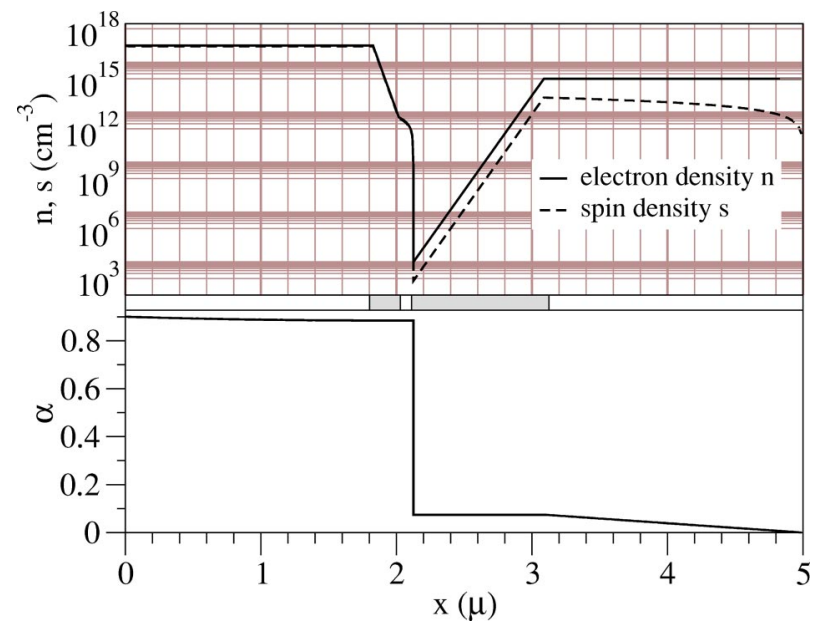

FIG. 4. Calculated electron- and spin-density profiles (top) and the spin polarization (bottom) in a nonmagnetic npn transistor with a source spin of polarization $\delta \alpha^{\text {source }}=0.9$ in the emitter. The densities inside the depletion layers (shaded boxes) are not calculated; they are shown, with no justification beside guiding the eye, as straight lines connecting the densities at the depletion layer edges. Bias voltages are $V_{b e}=0.5 \mathrm{~V}$ and $V_{b c}=0 \mathrm{~V}$. Somewhat less than $10 \%$ of the source spin polarization is transferred to the emitter.

The general formula for the spin injection, combining the processes (i) through (iii) in a magnetic transistor follows from the magnetic $p-n$ junction equations:

$$
\delta \alpha_{c}=\delta \alpha^{\text {source }} \gamma_{0, b e} \gamma_{1, b c} \frac{n_{0 b} e^{q V_{b e} / k_{B} T}}{N_{d c}} \frac{1-\alpha_{0 b} \alpha_{0 e}}{1-\alpha_{0 e}^{2}} .
$$

In the small injection limit $n_{0 b} \exp \left(q V_{b e} / k_{B} T\right) / N_{d c}$ is small (less than about 0.1 ); $\gamma_{0, b e}$ is of order 1 . The spin injection efficiency increases with increasing $\gamma_{1, b c}$. In the thin base limit and for a wide collector $\left(w_{c} \gg L_{s c}\right)$, for example, obtains $\gamma_{1, b c} \approx L_{s c} / w_{b}$. This can be as large as a 10 or a 100 , making $\alpha_{c}$ a significant fraction of $\alpha_{e} \approx \alpha^{\text {source }}$. The decrease of $w_{b}$ can be achieved by increasing the width of the $b-c$ depletion layer, which, in turn, increases with increasing $\left|V_{b c}\right|$.

Figure 4 illustrates the electrical spin injection of the source spin in our numerical model system using the full theory. The source spin of polarization $\delta \alpha^{\text {source }} \approx 0.9$ first diffuses towards the base with a small decrease due to spin relaxation. The spin polarization remains a constant through the $b-e$ depletion layer, resulting in a nonequilibrium spin density in the base. The spin polarization remains steady in the base, while both $n$ and $s$ decrease to their equilibrium values in going towards the $b-c$ depletion layer. Right before the depletion layer the spin polarization sharply drops, to get equal with $\alpha_{c} \approx 0.075$. Such sharp drops are characteristic of the spin pumping by the minority carriers. ${ }^{45}$

\section{B. Equilibrium spin}

Is there a way to accumulate spin in a MBT without first injecting a source spin into the structure? The answer is positive. In fact, there are two different nonequilibrium spin den- 
sities accumulating as a result of the carrier transport through the magnetic base. The first results from the spin extraction, acting in the emitter, the second from the intrinsic spin injection, effective in the collector.

The extracted spin accumulates in a way similar to the magnetic diode. ${ }^{45,51}$ The extracted spin density is small, on the order of the excess minority carrier densities. It is necessary that the base has an equilibrium spin polarization. The emitter can, but need not, be magnetic. Following Eq. (1) we get

$$
\delta s_{e}=-\gamma_{2, b e} \cosh \left(w_{b} / L_{n b}\right) s_{0 b} \exp \left(q V_{b e} / k_{B} T\right) .
$$

The result is a spin extraction from the emitter, since the accumulated excess spin $\delta s_{e}$ has the opposite sign than the equilibrium spin in the base. The extracted spin polarization $\delta \alpha_{e}=\delta s_{e} / N_{d e}$ is small due to the generally large value of $N_{d e}$. This extracted spin density can also be treated as the spin source which propagates to the emitter region, but the contribution to the collector spin is negligible, being of the order of $\left[n_{0 b} \exp \left(q V_{b e} / k_{B} T\right)\right]^{2} / N_{d e} N_{d c}$.

Intrinsic spin injection has no analog in the magnetic diode. The following physical processes are at work.

(i) Minority electron injection into the base. The base has a spin-split conduction band, so the electrons with the preferred spin will move at a faster rate, resulting in a nonequilibrium electron minority population, but with the equilibrium spin polarization. The spin density is then out of equilibrium.

(ii) The nonequilibrium spin density at $b e$ acts as a spin source in the $b-c$ junction, similar to the spin-polarized solar cell. $^{52}$

(iii) This "source" spin is injected into the collector, where it accumulates.

The result of the intrinsic spin injection, again in the limit of the thin base, is

$$
\delta \alpha_{c}=\alpha_{0 b} \gamma_{0, b e} \gamma_{1, b c} \frac{n_{0 b} e^{q V_{b e} / k_{B} T}}{N_{d c}} .
$$

If both $\alpha^{\text {source }}, \alpha_{0 b} \neq 0$ the total spin polarization in the collector is given by the sum of Eqs. (12) and (14). Remarkably, for $\alpha_{0 e}=0$, the equilibrium spin polarization $\alpha_{0 b}$ in Eq. (14) plays the role of $\delta \alpha_{e}$ in Eq. (14). The equilibrium spin polarization behaves, in MBT's, as a nonequilibrium source spin. This follows from the spin-selective electrical injection across the $b-e$ depletion layer.

Spin extraction and intrinsic electrical spin injection through a MBT are illustrated in Fig. 5 using the full theory. The equilibrium polarization spin in the base is $\alpha_{0 b}$ $=0.762$. The electrical transport through the base leads to a spin extraction from the emitter, with the extracted spin polarization $\alpha_{e} \approx-0.001$, small due to the large value of $N_{d e}$. The spin polarization jumps to its equilibrium value in the base, increasing sharply (see the discussion to Fig. 4) to $\alpha_{0 b}+\delta \alpha_{c}$ right before reaching the second depletion layer. The injected spin polarization is $\delta \alpha_{c} \approx 10 \%$, relatively large due to the small value of $N_{d c}$ and the large ratio $L_{s c} / w_{b}$ [see Eq. (12)].

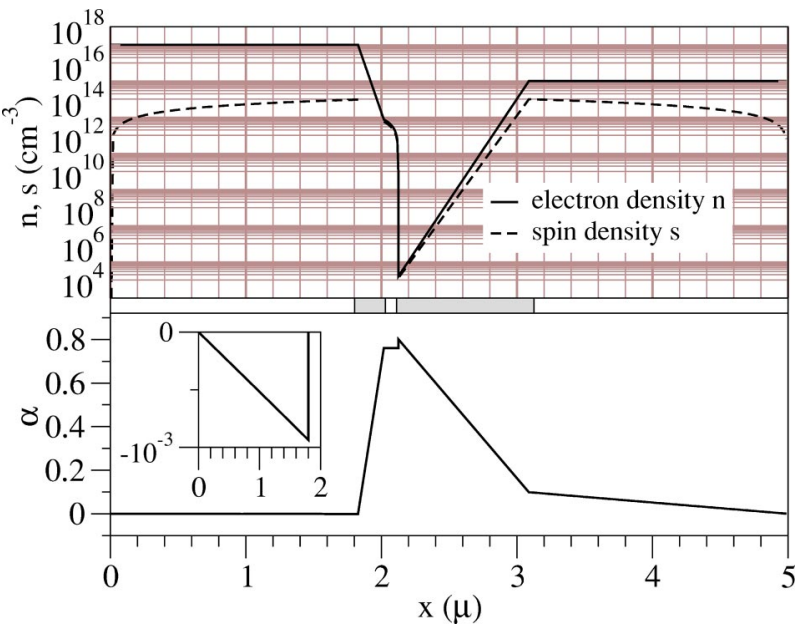

FIG. 5. Spin extraction and intrinsic spin injection. Calculated electron- and spin-density profiles (top) and the spin polarization (bottom) in an $n p n$ transistor with a magnetic base and nonmagnetic emitter and collector. In the emitter region spin density $s<0$ (extraction), here plotted as positive $(s \rightarrow-s)$; the spin density is not shown in the $b-e$ depletion layer. The equilibrium spin polarization in the base is $\alpha_{0 b}=0.762$, corresponding to the conduction-band splitting of $1 k_{B} T$. The bias voltages are $V_{b e}=0.5 \mathrm{~V}$ and $V_{b c}$ $=0 \mathrm{~V}$. The intrinsic spin injection, acting under the low-injection limit, results in the spin polarization in the collector of $\delta \alpha_{c}$ $\approx 10 \%$.

We expect that both the direct injection of the source spin as well as the spin extraction and the intrinsic spin injection become more efficient in the limits of large carrier injection (large biases), where our theory does not apply. This expectation is based on the results of numerical calculations ${ }^{51}$ of spin injection in magnetic diodes.

\section{ELECTRICAL CHARACTERISTICS}

The electrical properties of MBT's are determined by both the charge and the spin of the current carriers. There are two ways spin affects the electrical currents: through the dependence of the equilibrium minority electron and hole densities on the equilibrium spin polarization, and through the spincharge coupling resulting from the presence of a nonequilibrium spin. We first introduce the formalism for calculating electrical currents in bipolar transistors and then analyze the two ways in detail. We conclude with a discussion of the spin current through MBT's.

The scheme and the sign convention for the currents is shown in Fig. 6 (see also Fig. 1 for the description of symbols labeling the regions). Below we summarize the expressions for the currents from the Shockley theory of bipolar transistors. ${ }^{25,33}$ We write the expressions in a rather general form which turns out to be applicable also to MBT's (this follows from the generalized Shockley theory of magnetic diodes $\left.{ }^{45}\right)$. Let us define the generation current of a carrier $c$ (electron or hole) in region $r$ (emitter, base, or collector) as

$$
j_{g r}^{c}=\frac{q D_{c r}}{L_{c r}} c_{0 r} \operatorname{coth}\left(\frac{w_{r}}{L_{c r}}\right) .
$$




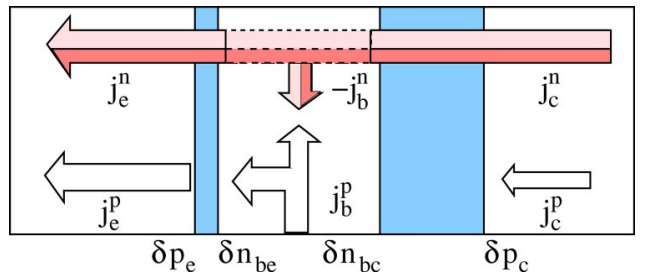

FIG. 6. Charge currents in an npn MBT. The electron emitter $j_{e}^{n}$ and collector $j_{c}^{n}$ currents are determined by the excess electron densities $\delta n_{b e}$ and $\delta n_{b c}$ in the base. Similarly, the hole emitter $j_{e}^{p}$ (collector $j_{c}^{p}$ ) current is determined by $\delta p_{e}\left(\delta p_{c}\right)$. The base current $j_{b}=j_{e}-j_{c}$ is formed by the electrons recombining with holes $\left(j_{b}^{n}\right)$ and by the holes that both recombine with electrons and enter the base from the base electrode $\left(j_{b}^{p}\right)$. Shading on the arrows of the electron currents indicate that the current is spin polarized.

The electron charge current density in the emitter is

$$
j_{e}^{n}=j_{g b}^{n}\left[\frac{\delta n_{b e}}{n_{0 b}}-\frac{1}{\cosh \left(w_{b} / L_{n b}\right)} \frac{\delta n_{b c}}{n_{0 b}}\right] .
$$

The first term in Eq. (16) represents the diffusion of electrons in the base at the $b-e$ junction. The second term describes a competing diffusion from the excess minority electrons at the $b-e$ junction. Through the current continuity, this base diffusion current continues to the emitter to become $j_{e}^{n}$. Similar expression holds for the electron current in the collector:

$$
j_{c}^{n}=j_{g b}^{n}\left[-\frac{\delta n_{b c}}{n_{0 b}}+\frac{1}{\cosh \left(w_{b} / L_{n b}\right)} \frac{\delta n_{b e}}{n_{0 b}}\right] .
$$

All the carrier densities appearing in the expressions for the currents can be calculated from the theory in Sec. III. Holes contribute to the currents through the diffusion of their excess minority populations $\delta p_{e}$ and $\delta p_{c}$. The expression for the densities is similar to Eq. (9) with no spin. For example,

$$
\delta p_{e}=p_{0 e}\left(e^{q V_{b e} / k_{B} T}-1\right) .
$$

The two hole currents are

$$
\begin{gathered}
j_{e}^{p}=j_{g e}^{p} \frac{\delta p_{e b}}{p_{0 e}}, \\
j_{c}^{p}=-j_{g c}^{p} \frac{\delta p_{c b}}{p_{0 c}} .
\end{gathered}
$$

The total emitter current is $j_{e}=j_{e}^{n}+j_{e}^{p}$, and similarly the total collector current is $j_{c}=j_{c}^{n}+j_{c}^{p}$. Finally, the base current is $j_{b}=j_{e}-j_{c}$. The task of computing the currents through an MBT is reduced to the computation of the excess electron and hole densities at the two depletion layers.

The current amplification coefficient (gain) $\beta$ is defined as

$$
\beta=\frac{j_{c}}{j_{b}} .
$$

If $\beta$ is large, small changes in $j_{b}$ lead to large variations in $j_{c}$, allowing signal amplification. The gain is often written as $^{33}$

$$
\beta=1 /\left(\alpha_{T}^{\prime}+\gamma^{\prime}\right) .
$$

Here $\alpha_{T}^{\prime}$ measures the (in)efficiency of the electron-hole recombination in the base, and $\gamma^{\prime}$ describes the (in)efficiency of the emitter electron injection into the base. The usual base transport factor ${ }^{33}$ is defined as $\alpha_{T}=1 /\left(1+\alpha_{T}^{\prime}\right)$ and the emitter efficiency factor as $\gamma=1 /\left(1+\gamma^{\prime}\right)$.

After substituting for the currents, the emitter (in) efficiency $\alpha_{T}^{\prime}$ is calculated to be

$$
\alpha_{T}^{\prime}=\cosh \left(\frac{w_{b}}{L_{n b}}\right)-1
$$

This is the value obtained for conventional transistors and is also valid for MBT's. Coefficient $\alpha_{T}^{\prime}$ does not depend on spin since it reflects only the electron-hole recombination in the base and in our model the recombination is spin independent. However, there may be cases where $L_{n b}$ depends significantly on $\alpha_{0 b}$, in which case the gain could be controlled by spin even through $\alpha_{T}^{\prime}$. Note that $w_{b}$ depends on the equilibrium spin through the spin dependence of the built-in fields. ${ }^{45}$ Equation (23) holds even for such cases. On the other hand, $\gamma^{\prime}$ depends explicitly on both the equilibrium and nonequilibrium spin. We describe this dependence by defining a new parameter $\eta$ :

$$
\gamma^{\prime}=\gamma_{0}^{\prime} / \eta
$$

where $\gamma_{0}^{\prime}$ is the emitter efficiency of a conventional npn transistor $^{33}$

$$
\gamma_{0}^{\prime}=\frac{N_{a b} D_{p e} n_{i e}^{2}}{N_{d e} D_{n b} n_{i b}^{2}} \frac{L_{n b} \sinh \left(w_{b} / L_{n b}\right)}{L_{p e} \tanh \left(w_{e} / L_{p e}\right)},
$$

where we allow for a generally different intrinsic carrier concentrations $n_{i e}$ and $n_{i b}$ in the emitter and base, respectively. In the following two sections we discuss the physics behind $\eta$, which we call the magnetoamplification coefficient. We will, in particular, consider the thin base limit, where $\alpha_{T}^{\prime}$ $\sim\left(w_{b} / L_{n b}\right)^{2}, \gamma_{0}^{\prime} \sim\left(w_{b} / L_{n b}\right)$, and $\gamma_{0}^{\prime}$ dominates the current amplification (for example, in $\mathrm{Si}$ ). In such cases

$$
\beta \approx \eta / \gamma_{0}^{\prime} .
$$

If the base transport factor is not negligible, the spin control efficiency diminishes.

\section{A. Magnetoamplification effect: Influence of the equilibrium spin}

Consider a magnetic base. The Boltzmann statistics gives $^{45,51}$

$$
n_{0 b}=\frac{n_{i b}^{2}}{N_{a b}} \tanh \left(q \zeta_{b} / k_{B} T\right)=\frac{n_{i}^{2}}{N_{a b}} \frac{1}{\sqrt{1-\alpha_{0 b}^{2}}} .
$$

Since $j_{g b}^{n} \sim n_{0 b}$ [see Eq. (15)], it follows that the base generation current increases as $\alpha_{0 b}$ (that is, its magnitude) increases. In turn, $j_{e}^{n}, j_{c}^{n} \sim j_{g b}^{n}$, so that both the emitter and the collector currents increase with increasing $\alpha_{0 b}$. The equilib- 


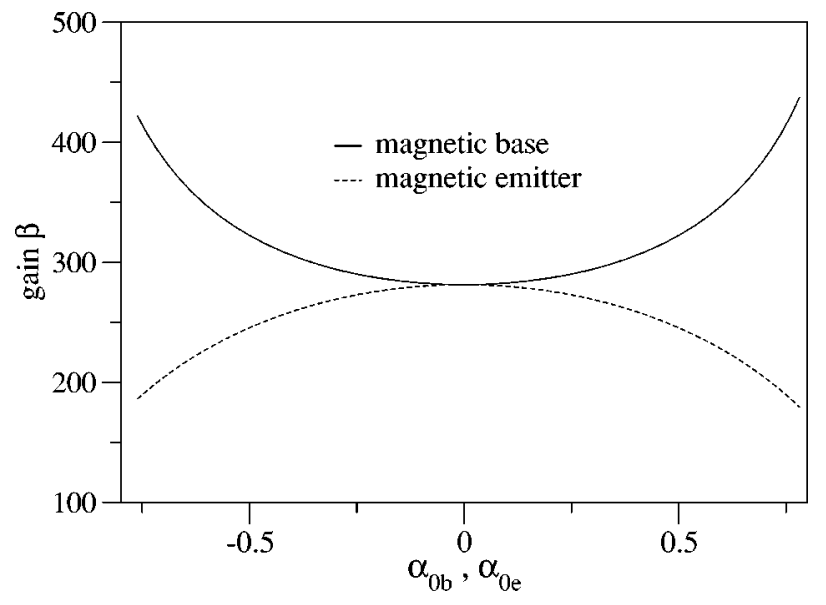

FIG. 7. Calculated gain $\beta$ of a MBT with a magnetic base (solid) and emitter (dashed) for our numerical model. No source spin is present. The equilibrium base (emitter) spin polarization is $\alpha_{0 b}\left(\alpha_{0 e}\right)$. The calculation is done on a structure with the nominal base width of $1.5 \mu \mathrm{m}$ to keep the effective width $w_{b}$ positive for the considered range of polarizations.

rium spin controls the charge currents flowing in MBT, leading to a magnetoresistance effect. Spin-unpolarized holes too contribute to the spin control of the currents, as shown by Lebedeva and Kuivalainen ${ }^{31}$ for a pnp MBT. If the emitter is magnetic, the minority hole density is

$$
p_{0 e}=\frac{n_{i e}^{2}}{N_{d c}} \frac{1}{\sqrt{1-\alpha_{0 e}^{2}}},
$$

analogously for the collector (in the amplification mode the hole density in the collector is negligible and does not affect the current properties). The hole emitter current increases with increasing $\alpha_{0 e}$.

The magnetoamplification coefficient amounts to the simple expression

$$
\eta=\sqrt{\frac{1-\alpha_{0 e}^{2}}{1-\alpha_{0 b}^{2}}},
$$

and in the thin base limit, Eq. (26) is thus described by the gain

$$
\beta=\frac{1}{\gamma_{0}^{\prime}} \sqrt{\frac{1-\alpha_{0 e}^{2}}{1-\alpha_{0 b}^{2}}} .
$$

The gain of MBT's can thus be controlled by controlling the equilibrium magnetization (for example, by changing the external magnetic field) of the emitter or the base. The collector magnetization plays no role. On the other hand, the two equilibrium polarizations $\alpha_{0 e}$ and $\alpha_{0 b}$ act against each other: The gain increases (decreases) with a greater spin splitting in the base (emitter). This is because the emitter (in)efficiency $\gamma^{\prime}$ increases (decreases) if there are relatively more holes (electrons) present in $j_{e}$. If the spin polarization is uniform across the $b-e$ junction, the gain is spin independent.
The opposite role of the equilibrium magnetizations in the base and in the emitter is shown in Fig. 7, which illustrates the behavior of $\beta$ with respect to the changes of $\alpha_{0 b}$ and $\alpha_{0 e}$ separately. The calculation is done using the full theory, not the approximate formulas above. However, the approximation describes the calculation very well, showing that spindependent effects, for example, on the effective widths $w$, which are accounted for in the full theory, play minor role in our example.

\section{B. Giant magnetoamplification effect: Spin-charge coupling in MBT}

A nontrivial realization of the Silsbee-Johnson spincharge coupling ${ }^{46,47}$ representing the physics of the proximity of an equilibrium and nonequilibrium spin in MBT's is what we call here the giant magnetoamplification effect (GMA), in analogy with giant magnetoresistance (GMR) effect in metallic multilayers. ${ }^{53}$ For GMA it is necessary that there be a nonequilibrium spin polarization in the emitter (arising from a source spin) and an equilibrium spin either in the base or in the emitter (or both). The physics is illustrated in Fig. 2. The charge current through MBT's depends on the relative orientation of the source and the equilibrium spins, because of the spin-dependent barrier in the $b$-e junction.

In the presence of a nonequilibrium spin density $\delta \alpha_{e}$, the magnetoamplification coefficient becomes

$$
\eta=\sqrt{\frac{1-\alpha_{0 e}^{2}}{1-\alpha_{0 b}^{2}}}\left[1+\delta \alpha_{e}\left(\alpha_{0 b}-\alpha_{0 e}\right) /\left(1-\alpha_{0 e}\right)^{2}\right] .
$$

If only the base is magnetic, the gain in the thin base limit is

$$
\beta=\frac{1}{\gamma_{0}^{\prime}} \frac{1+\delta \alpha_{e} \alpha_{0 b}}{\sqrt{1-\alpha_{0 b}^{2}}} .
$$

The spin-charge coupling is described by the product $\delta \alpha_{e} \alpha_{0 b}$, similar to implications of the spin-voltaic effect in magnetic $p$-n junctions. ${ }^{45,51,54}$ Let $\beta_{\max }$ and $\beta_{\min }$ are the gains for the configuration of the source and equilibrium spins (parallel or antiparallel) that yield the maximum and minimum gain, respectively. For a magnetic base (emitter) the maximum is achieved at parallel (antiparallel) orientation and the minimum at antiparallel (parallel) orientation of the source and equilibrium spins, respectively. We define the GMA coefficient as

$$
\mathrm{GMA}=\frac{\beta_{\max }-\beta_{\min }}{\beta_{\min }},
$$

in analogy with a similar expression (involving resistivities) defining the GMR coefficient.

For the magnetic base

$$
\mathrm{GMA}=\frac{2\left|\delta \alpha_{e} \alpha_{0 b}\right|}{1-\left|\delta \alpha_{e} \alpha_{0 b}\right|} .
$$

If, for example, $\delta \alpha_{e}=\alpha_{0 b}=0.5, \mathrm{GMA}=67 \%$. The analogy with GMR is clear: there is a large magnetoresistance effect (greater than 10\%), which is most pronounced when the rela- 


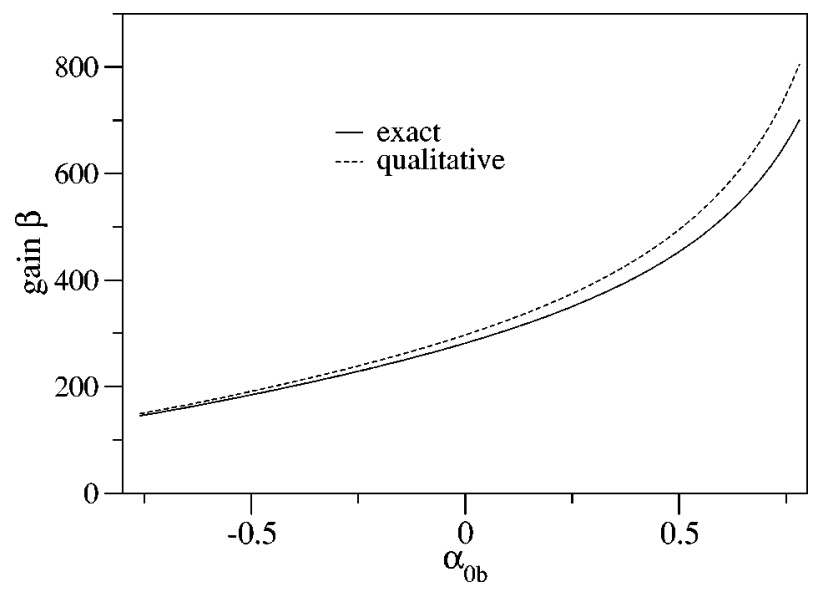

FIG. 8. Giant magnetoamplification effect. Calculated gain $\beta$ of our MBT example with a magnetic base and source emitter spin, as a function of $\alpha_{0 b}$ for a fixed $\alpha^{\text {source }}=0.9$. The solid curve is the calculation using the full theory, the dashed curve is the contribution of the emitter efficiency only, $\beta \approx \eta / \gamma^{\prime}$, where the magnetoamplification coefficient $\eta$ is given by Eq. (31). The calculations are done on a structure with the base $1.5 \mu \mathrm{m}$ long, as in Fig. 7.

tive orientation of two spin polarizations changes from parallel to antiparallel. If, on the other hand, the emitter is magnetic, the effect is opposite: the parallel spin orientation decreases the gain, due to the decrease in the emitter injection efficiency. The GMA coefficient is

$$
\mathrm{GMA}=\frac{2\left|\delta \alpha_{e} \alpha_{0 e}\right|}{1-\alpha_{0 e}^{2}+\left|\delta \alpha_{e} \alpha_{0 e}\right|}
$$

The GMA coefficient vanishes if $\alpha_{0 e}=\alpha_{0 b}$. To decide on whether to use a magnetic base or a magnetic emitter one needs to take into account that a magnetic base will have a smaller $L_{s b}$. If $L_{s b} \lesssim w_{b}$, a magnetic emitter would be instead preferable. Note that the GMA coefficient is directly proportional to the magnitude of $\delta \alpha_{e}$, and so it can be used to measure the nonequilibrium spin polarization, as in the case of magnetic diodes. ${ }^{55}$

Figure 8 illustrates GMA on our MBT example with a magnetic base and source spin polarization $\alpha^{\text {source }}=0.9$ (as in Fig. 2). The solid line represents the calculation of $\beta\left(\alpha_{0 b}\right)$ using the full theory, while the dashed line is the approximation, Eq. (32), valid for the thin base transistors. The approximative formula works very well. The asymmetric curve demonstrates the GMA effect. When the equilibrium and the source spin are antiparallel, $\beta$ is small; when they are parallel, $\beta$ is large. The magnetic control of the charge current and its amplification is thus predicted to be rather effective.

\section{Spin-polarized current}

Thus far we have studied the spin polarization of the electron density as a measure of the spin injection efficiency. This spin polarization is typically detected by optical experiments. Another spin polarization, that of the charge current, is more convenient for theory and is invariably used in spin injection models to assess the spin injection efficiency (see the $\gamma$ technique of Rashba ${ }^{56,57}$ ) and to establish the boundary conditions for spin at the interfaces. ${ }^{45}$ The current spin polarization $\alpha_{j}$ is the ratio of the spin current $j_{s}$, which is the difference between the charge currents formed by the spin-up and spin-down electrons, and the total charge current $j: \alpha_{j}$ $=j_{s} / j$. The current spin polarization is much less intuitive than the density spin polarization. There are several reasons for that. First, $\alpha_{j}$ involves not only the carrier and spin densities, but also the drift or diffusion velocities which can be spin dependent. Next, unlike $\alpha$, the magnitude of $\alpha_{j}$ is not restricted to the interval $(0,1)$. The magnitude of $\alpha_{j}$ is not even bound. Indeed, the spin current can be finite even if there is no charge current, making $\alpha_{j}$ infinite. Also the signs of $\alpha_{j}$ and $\alpha$ can be different. Finally, unlike the charge current, the spin current need not be uniform. Because of spin relaxation, $j_{s}$ (and also $\alpha_{j}$ ) is not conserved. For the above reasons, unless the relation between $\alpha$ and $\alpha_{j}$ is obvious or is explicitly derived, $\alpha_{j}$ is not indicative of the spin injection efficiency. In particular, in inhomogeneous (or hybrid) semiconductors at degenerate doping densities or at large biases, both spin diffusion and spin drift are relevant and one needs to employ the Poisson equation to solve the transport problem self-consistently to obtain a dependence between $\alpha$ and $\alpha_{j}$. Many of the experimental spin injection results are likely to fall in this category, making realistic theoretical modeling difficult.

Fortunately, in the low-injection limit there is a simple relation between the spin current and the spin, so the knowledge of $\alpha_{j}$ together with the knowledge of the charge current $j$ suffice to obtain $\alpha$. The spin current density at point $c$ (see Fig. 1) is readily obtained from Table II in Ref. 45:

$$
j_{s c}=\frac{q D_{n c}}{L_{s c}} \operatorname{coth}\left(w_{c} / L_{s c}\right) \delta s_{c} .
$$

Thus, $\delta \alpha_{c}=\delta s_{c} / N_{d c}$ is directly proportional to $j_{s c}=j \alpha_{j}$. As is shown below, $\alpha_{j}$ is usually comparable to $\alpha_{0}$ or $\delta \alpha$, largely independent on the biases. The spin injection efficiency is then determined by $j_{c}$, which, in turn, depends exponentially on $V_{b e}$.

We adopt the same sign convention for the spin currents as for the charge currents, see Fig. 6. A straightforward application of the magnetic $p-n$ junction equations (Sec. III) gives

$$
\alpha_{j c}=\frac{e^{q V_{b e} / k_{B} T}\left(\alpha_{0 b}+\delta \alpha_{e} \frac{1-\alpha_{0 b} \alpha_{0 e}}{1-\alpha_{0 e}^{2}}\right)-\alpha_{0 b}}{e^{q V_{b e} / k_{B} T}\left(1+\delta \alpha_{e} \frac{\alpha_{0 b}-\alpha_{0 e}}{1-\alpha_{0 e}^{2}}\right)-1} .
$$

What is striking (although not so surprising) is that $\alpha_{j c}$ in the cases of practical biases $\left|V_{b e}\right| \gg k_{B} T$ is bias independent. Indeed, for a forward bias $V_{b e}$, that is, in the amplification mode,

$$
\alpha_{j c}=\frac{\alpha_{0 b}\left(1-\alpha_{0 e}^{2}\right)+\delta \alpha_{e}\left(1-\alpha_{0 b} \alpha_{0 e}\right)}{1-\alpha_{0 e}^{2}+\delta \alpha_{e}\left(\alpha_{0 b}-\alpha_{0 e}\right)} .
$$




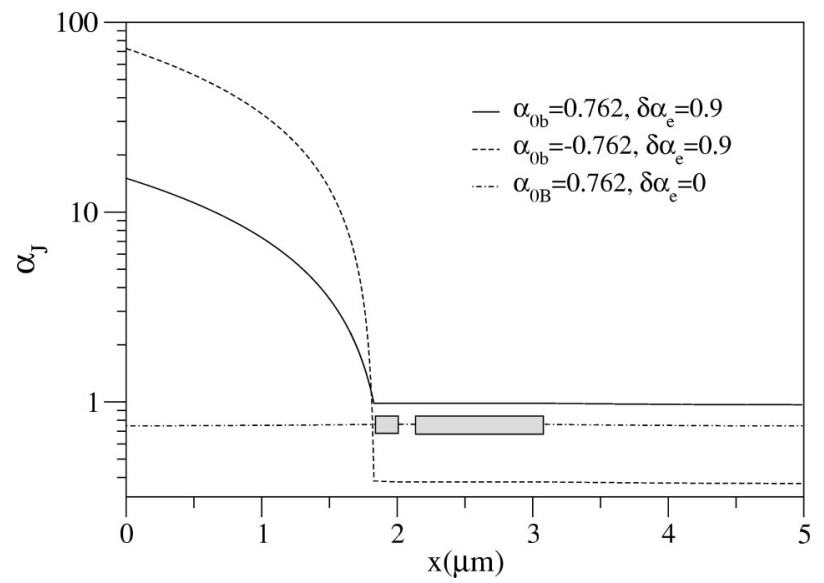

FIG. 9. Calculated spin current polarization profile in the magnetic npn transistor, calculating by the full theory. The source spin at the emitter is fixed at $\delta \alpha^{\text {source }}=0.9$, while the equilibrium base spin polarization changes sign at the magnitude of $\alpha_{0 b}=0.762$, corresponding to the base spin splitting of the order of the thermal energy. The biases are $V_{b e}=0.5 \mathrm{~V}$ and $V_{b c}=0 \mathrm{~V}$. The spin current remains uniform across the depletion layer, based on the assumption of negligible spin relaxation there. The spin current is normalized by the emitter and the collector currents in the emitter and the collector regions, and by the base electron current in the base. The horizontal line is for no source spin. The spin current stays uniform throughout the sample at the value given by $\alpha_{0 b}$, implying no significant spin relaxation, but not necessarily spin injection.

This is in sharp contrast, for example, to Eq. (12) which displays the exponential increase of the spin injection efficiency with $V_{b e}$. In the limit of no source spin, Eq. (38) reduces to $\alpha_{j c}=\alpha_{0 b}$, the main result of Flatte et al. ${ }^{30}$ Similarly, if the transistor has no equilibrium spin, the spin current polarization is $\alpha_{j c}=\delta \alpha_{e}$. If the emitter/base bias is reverse, $V_{b e}<0$, spin injection is practically nonexisting. Yet, there is a large current spin polarization, $\alpha_{j c}=\alpha_{0 b}$, independent of the source spin, confirming our claim that large $\alpha_{j}$ alone does not imply efficient spin injection.

Our analysis is illustrated in Fig. 9. We consider the amplification mode, but the results vary little with the applied bias, as explained above. Figure 9 describes three cases. One with the source spin and the equilibrium spin pointing in the same direction, one where the two spins are antiparallel, and one where only the equilibrium spin is present. In all the cases $\alpha_{j c}$ (which is the value at $x \approx 3 \mu \mathrm{m}$ ) is smaller than 1 , but rather considerable, consistent with Eq. (38). Note that $\alpha_{j}$ in the emitter is much greater than 1 for the case of the source spin, due to the spin-diffusion current being greater than the majority electron drift current there. The value of $\alpha_{j}$ decreases at the emitter/base junction, where it becomes a constant, signifying small spin relaxation in both the base and in the emitter. Finally, the case of no source spin polarization $\left(\delta \alpha_{e} \ll \alpha_{0 b}\right)$ shows a flat $\alpha_{j}$. This case, viewed from the density spin polarization perspective, is shown in Fig. 5. The nonequilibrium spin that accumulates in the base is injected to the collector, and extracted from the emitter. The injection/extraction efficiency increases exponentially with $V_{b e}$. For small biases, even if $\alpha_{j}$ is equal to $\alpha_{0 b} \approx 0.762$, the spin injection is negligible. Interestingly, even if the spin current polarization is positive in the emitter, the spin-density polarization is negative (spin extraction, cf. Fig. 5).

\section{CONCLUSIONS}

We have developed an analytic theory for the spinpolarized transport in magnetic bipolar transistors in the lowinjection regime. We have shown that the transistor displays a number of different phenomena, not observed either in the conventional spin-unpolarized bipolar transistor or in the magnetic diode. One such effect is the intrinsic electrical spin injection, which is a spin injection from a magnetic base into a nonmagnetic collector, without the presence of a source spin in the emitter. The spin injection efficiency depends exponentially on the emitter/base bias. Other effects are related to the gain which can be influenced in two ways. First, by the equilibrium spin, either in the emitter or in the base, modifying the generation current. Second, by the spincharge coupling, modifying directly the electron injection from the emitter.

Bipolar junction transistors are used for ultra-high-speed logic applications thanks to the fast carrier transport. Spin can bring additional functionalities. The magnetoamplification effects can be used to study spin signals (time varying spin polarizations) by detecting charge currents. Indeed, all the currents in a MBT in the amplification mode depend primarily on $\delta n_{b e}$, which is controlled by both the equilibrium and nonequilibrium spin. The spin-to-current conversion can thus be observed by measuring the collector current, or directly the GMA coefficient Eq. (33). On the other hand, the amount of the injected spin in the collector depends exponentially on $V_{b e}$ in the same manner as the charge current depends on it. Changes in, for example, the base current can thus lead to changes in the spin polarization $\delta \alpha_{c}$. As a result, current signals can be detected by observing the accumulated spin polarization. Perhaps the most attractive feature of the magnetoamplification effects is that the spin splitting or the source spin polarization are not built-in device properties but can change on demand, during the transistor operation, by magnetic field. This is why an MBT is an example of a variable heterostructure transistor.

Another use of MBT's may be in the electrically induced magnetization switching, similar to what has been observed as light-induced ferromagnetism ${ }^{13,14}$ or ferromagnetism induced by the gate voltage of field-effect transistors. ${ }^{15,16}$ If the base is a ferromagnetic semiconductor, the equilibrium magnetization depends on the density of free carriers. This density can be, in turn, controlled by $V_{b e}$. While the scenario of the electrically induced ferromagnetism in a nondegenerate MBT is probably not realistic, at higher doping and current injection levels (where our theory no longer applies) this effect could be observable.

We believe that the phenomena we propose to study are robust and should be observed. For the spin-source spin injection one does not need a magnetic semiconductor in the structure. The source spin can be generated in the emitter either optically or electrically, and similarly the spin injected into the collector can be observed by detecting the polarization of electroluminescence. ${ }^{4,6}$ For the phenomena related to 


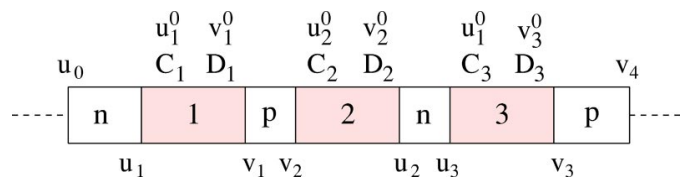

FIG. 10. Notation scheme for an array of $p-n$ junctions. The figure illustrates an $n p n p$ (thyristor) structure. The depletion layers are shaded. The known quantities are shown above the junctions, the unknown below. Each junction $i$ is characterized by the scalar $u_{i}^{0}$ and by the triplet of vectors $\mathbf{v}_{i}^{0}, \mathbf{C}_{i}$, and $\mathbf{D}_{i}$, which are determined by the doping densities and the equilibrium carrier and spin densities of the regions adjacent to the junction, and by the applied voltage $V_{i}$ across the junction. The nonequilibrium spin density $u_{0}$ (note that the symbol 0 here denotes the region, not an equilibrium) and the charge-/spin-density vector $\mathbf{v}_{4}$ would be the boundary conditions here. The densities $u_{i}$ and $\mathbf{v}_{i}$ at the depletion regions are to be obtained self-consistently. These densities are all what is needed to calculate the charge and spin currents.

the equilibrium magnetism, the rule of thumb is that the spin splitting should be comparable to the thermal energy in order to create noticeable spin polarizations. While to see GMA at room temperature may be difficult at present, at smaller temperatures (starting from $50 \mathrm{~K}$ where the shallow donors and acceptors start to ionize) the effects could be detected at the spin splitting levels of $\sim 1 \mathrm{meV}$.

\section{ACKNOWLEDGMENTS}

We are grateful to S. Das Sarma for support and encouragement, and for useful discussions. I.Ž. acknowledges the National Research Council for financial support. This work was funded by DARPA, the NSF-ECS, and the U.S. ONR.

\section{APPENDIX: AN ARRAY OF MAGNETIC $p-n$ JUNCTIONS}

We introduce a formalism for evaluating the carrier and spin densities in an array of magnetic $p-n$ junctions. An array of two junctions forms a MBT, while three junctions would form a magnetic thyristor. The boundary conditions are applied for the densities; it is straightforward to adapt the method to have boundary conditions specified by the spin currents. All the junctions need to be considered simultaneously since there is both intrajunction and interjunction charge and spin coupling. The intrajunction coupling arises from the generalized Shockley conditions ${ }^{45}$ of the uniformity of the spin-resolved chemical potentials and of the continuity of the charge and spin currents, across a junction's depletion layer. The interjunction coupling arises from the carrier and spin diffusion in the bulk regions between the depletion layers: the carrier and spin density at one end influences the current at the other end, and vice versa. The two couplings lead to a set of linear algebraic equations for the densities.

The array we consider is shown in Fig. 10. Each junction is given a number $i$ starting from one. The junctions are either of the $p-n$ ( $p$ left and $n$ right) or of the $n-p$ ( $n$ left and $p$ right) type. In the following the indices $n$ and $p$ relate to the $n$ and $p$ regions adjacent to the junction in question. Let us introduce the notation using a generic junction, as in Sec. III. The bias $V$ across the junction is positive for the forward and negative for the reverse direction of the charge current. The electron density is $n$, with index zero $\left(n_{0}\right)$ reserved for the equilibrium value. The nonequilibrium (excess) part of the density is denoted as $\delta n=n-n_{0}$. Similarly for the electron-spin density $s$ and for the spin polarization $\alpha$ $=s / n$. The doping densities are $N_{d}$ for the donors in the $n$ region and $N_{a}$ for the acceptors in the $p$ region.

We make the complex notation more compact by introducing some unifying symbols. We first denote by $u^{0}$ the scalar characterizing the nonequilibrium spin density due to the carrier extraction:

$$
u^{0}=-\gamma_{2} \cosh \left(w_{p} / L_{n p}\right) s_{0 p}\left(e^{q V / k_{B} T}-1\right) .
$$

Here $k_{B} T$ is the thermal energy, with $T$ denoting temperature; $q$ is the proton charge. Parameters $\gamma$ are introduced in Eqs. (3)-(5). To properly account for the spin current in the $n$ region, one needs to apply the rescaling in Eq. (6). We also introduce vector $\mathbf{v}^{0}$ which specifies the nonequilibrium electron and spin density in the $p$ region as a result of the carrier injection (we stress that the upper indices do not indicate the equilibrium values):

$$
\mathbf{v}^{0}=\left(e^{q V / k_{B} T}-1\right)\left[n_{0 p}, s_{0 p}\right] .
$$

The dimensionless vector $\mathbf{C}$ characterizes intrajunction coupling:

$$
\mathbf{C}=\left[\alpha_{0 p}\left(\gamma_{2}-\gamma_{1}\right), \gamma_{1}\right]
$$

while another dimensionless vector $\mathbf{D}$, given by

$$
\mathbf{D}=\frac{n_{0 p}}{N_{d}} \frac{e^{q V / k_{B} T}}{1-\alpha_{0 n}^{2}}\left[\alpha_{0 p}-\alpha_{0 n}, 1-\alpha_{0 p} \alpha_{0 n}\right],
$$

characterizes interjunction coupling. The quantities $u^{0}, \mathbf{v}^{0}$, $\mathbf{C}$, and $\mathbf{D}$ are presumed to be known. They are the input parameters.

The unknown quantities are the nonequilibrium carrier and spin densities at the depletion layers. In the low-bias regime considered here, the electron density in the $n$ regions is fixed: $n_{n} \approx N_{d}$, and only $\delta s_{n}$ needs to be calculated. In the $p$ regions the excess electron (spin) density $\delta n_{p}\left(\delta s_{p}\right)$ is unknown. We denote by scalar $u$ the nonequilibrium spin density $\delta s_{n}$ in the adjacent $n$ region:

$$
u=\delta s_{n} .
$$

Vector $\mathbf{v}$ will describe both the nonequilibrium electron and spin density in the $p$ region:

$$
\mathbf{v}=\left[\delta n_{p}, \delta s_{p}\right] .
$$

The boundary conditions are given by the corresponding $u$ or $\mathbf{v}$ (depending on whether the $n$ or the $p$ region is the contact region) at the beginning and the end of the array.

Using the above notation, the magnetic $p-n$ junction equations, Eqs. (1), (9), and (10), are greatly simplified and can be adapted to solve the array problem. Indeed, for junction $i$ the $p-n$ junction equations can be written as

$$
u_{i}=u_{i}^{0}+\gamma_{0, i} u_{i \pm 1}+\mathbf{C}_{i} \cdot \mathbf{v}_{i \mp 1},
$$




$$
\mathbf{v}_{i}=\mathbf{v}_{i}^{0}+\mathbf{D}_{i} u_{i}
$$

where the upper (lower) sign is for the $p-n(n-p)$ directed junction. For the npn MBT described in the main text, the equations take the form

$$
\begin{gathered}
u_{1}=u_{1}^{0}+\gamma_{0,1} u_{0}+\mathbf{C}_{1} \cdot \mathbf{v}_{2}, \\
\mathbf{v}_{1}=\mathbf{v}_{1}^{0}+\mathbf{D}_{1} u_{1},
\end{gathered}
$$

for junction 1 , and

$$
\begin{gathered}
u_{2}=u_{2}^{0} \gamma_{0,2} u_{3}+\mathbf{C}_{2} \cdot \mathbf{v}_{1}, \\
\mathbf{v}_{2}=\mathbf{v}_{2}^{0}+\mathbf{D}_{2} u_{2},
\end{gathered}
$$

for junction 2. Analogous equations can be written for holes. The solution to Eqs. (A9)-(A12) is

$$
u_{2}=\left(\mathbf{C}_{2} \cdot \mathbf{D}_{1}\right)\left(\gamma_{0,1} u_{0}+u_{1}^{0}\right)+\mathbf{C}_{2} \cdot \mathbf{v}_{1}^{0}+u_{2}^{0}+\gamma_{0,2} u_{3},
$$

where we have neglected the terms of order $\left[n_{0 p} \exp (V) /\right.$ $\left.N_{d}\right]^{2}$, small in the low-bias regime. The formulas for $u_{1}, \mathbf{v}_{1}$, and $\mathbf{v}_{2}$ can be obtained directly by substituting Eq. (A13) back to Eqs. (A9) through (A12).

Equation (A13) describes spin injection through a MBT, since $u_{2}$ is the nonequilibrium spin in the collector at the depletion layer with the base. The first term on the right-hand side of Eq. (A13) represents the transfer of source spin $u_{0}$ from the emitter to the collector. Indeed, for a nonmagnetic transistor (the equilibrium spin polarizations are zero) the transferred source spin is $u_{3}=\gamma_{0,1} \gamma_{1,2} n_{0 p} \exp \left(V_{1} / k_{B} T\right) u_{0}$. Here $\gamma_{0}$ describes the transfer of the source spin through the emitter-a majority carrier spin injection. Once the spin is in the base, it becomes the spin of the minority carriers [hence the minority density factor $n_{0 b} \exp \left(V_{1} / k_{B} T\right)$ ], diffusing towards the base/collector depletion layer 2 . The built-in elec- tric field in this layer sweeps the spin into the collector, where it becomes the spin of the majority carriers again, by the process of the minority carrier spin pumping. ${ }^{45,50}$ Can the injected spin polarization in the collector be greater than the source spin polarization? The answer is negative in the lowinjection regime. It would be tempting to let the spindiffusion length in the collector to increase to large values to get a greater pumped spin. But that would increase the importance of the electric field in the $n$ regions and the theory (which is based on the charge and spin diffusion and not on the spin drift) would cease to be valid. However, the spin density in the collector can be greater than that in the base, demonstrating that the spatial decay of the nonequilibrium spin (spin accumulation) is not, in general, monotonically decreasing, in line of what was demonstrated in nonmagnetic $p$ - $n$ junctions ${ }^{50}$ as well as in inhomogeneous unipolar semiconductors. ${ }^{58}$ The same term also describes the transfer of the nonequilibrium spin $u_{1}^{0}$ accumulated as a result of the electron injection into the magnetic base. The second term of Eq. (A13), which is independent of the source spin, results from the (intrinsic) spin pumping by the minority channel of the nonequilibrium spin generated in the base by the forward current through junction 1. This term vanishes if the base is nonmagnetic $\left(\alpha_{0 b}=0\right)$. The third term of Eq. (A13) represents the spin extraction due to the magnetic base. This term is controlled by $V_{2}$, the bias at the base/collector junction. For the reverse bias, used in the amplification mode, this term can be neglected. Finally, the last term of Eq. (A13) describes the diffusion of the source spin in the collector.

The knowledge of the carrier and spin densities at the depletion layers allows us to calculate the charge and spin currents in the systems, as well as the density spatial profiles throughout the bulk regions, using the formulas for magnetic $p-n$ junctions in Table II of Ref. 45. This is done for our numerical MBT model presented in Secs. IV and V.
*Present address: Center for Computational Materials Science, Naval Research Laboratory, Washington, D. C. 20735, USA.

${ }^{1}$ S. Das Sarma, J. Fabian, X. Hu, and I. Žutić, Solid State Commun. 119, 207 (2001).

${ }^{2}$ V.V. Osipov, N.A. Viglin, and A.A. Samokhvalov, Phys. Lett. A 247, 353 (1998).

${ }^{3}$ P.R. Hammar and M. Johnson, Phys. Rev. Lett. 88, 066806 (2002).

${ }^{4}$ R. Fiederling, M. Kleim, G. Reuscher, W. Ossau, G. Schmidt, A. Waag, and L.W. Molenkamp, Nature (London) 402, 787 (1999).

${ }^{5}$ Y. Ohno, D.K. Young, B. Beschoten, F. Matsukura, H. Ohno, and D.D. Awschalom, Nature (London) 402, 790 (1999).

${ }^{6}$ B.T. Jonker, Y.D. Park, B.R. Bennett, H.D. Cheong, G. Kioseoglou, and A. Petrou, Phys. Rev. B 62, 8180 (2000).

${ }^{7}$ H. Munekata, H. Ohno, R.R. Ruf, R.J. Gambino, and L.L. Chang, J. Cryst. Growth 111, 1011 (1991).

${ }^{8}$ H. Ohno, Science 281, 951 (1998).

${ }^{9}$ L. Esaki, P. Stiles, and S. von Molnár, Phys. Rev. Lett. 19, 852 (1967)

${ }^{10}$ X. Hao, J.S. Moodera, and R. Meservey, Phys. Rev. B 42, 8235 (1990)
${ }^{11}$ J.M. Kikkawa and D.D. Awschalom, Phys. Rev. Lett. 80, 4313 (1998).

${ }^{12}$ R.I. Dzhioev, V.L. Korenev, I.A. Merkulov, B.P. Zakharchenya, D. Gammon, A.L. Efros, and D.S. Katzer, Phys. Rev. Lett. 88, 256801 (2002).

${ }^{13}$ S. Koshihara, A. Oiwa, M. Hirasawa, S. Katsumoto, Y. Iye, S. Urano, H. Takagi, and H. Munekata, Phys. Rev. Lett. 78, 4617 (1997).

${ }^{14}$ A. Oiwa, Y. Mitsumori, R. Moriya, T. Supinski, and H. Munekata, Phys. Rev. Lett. 88, 137202 (2002).

${ }^{15}$ H. Ohno, D. Chiba, F. Matsukura, T.O.E. Abe, T. Dietl, Y. Ohno, and K. Ohtani, Nature (London) 408, 944 (2000).

${ }^{16}$ Y.D. Park, A.T. Hanbicki, S.C. Erwin, C.S. Hellberg, J.M. Sullivan, J.E. Mattson, T.F. Ambrose, A. Wilson, G. Spanos, and B.T. Jonker, Science 295, 651 (2002).

${ }^{17}$ G. Schmidt, G. Richter, P. Grabs, C. Gould, D. Ferrand, and L.W. Molenkamp, Phys. Rev. Lett. 87, 227203 (2001).

${ }^{18}$ S.D. Ganichev, E.L. Ivchenko, V.V. Belkov, S.A. Tarasenko, M. Sollinger, D. Weiss, W. Wegschelder, and W. Prettl, Nature (London) 417, 153 (2002).

${ }^{19}$ D.J. Monsma, J.C. Lodder, T.J.A. Popma, and B. Dieny, Phys. Rev. Lett. 74, 5260 (1995). 
${ }^{20}$ S. van Dijken, X. Jiang, and S.S.P. Parkin, Phys. Rev. Lett. 90, 197203 (2003).

${ }^{21}$ X. Jiang, R. Wang, S. van Dijken, R. Shelby, R. Macfarlane, G.S. Solomon, J. Harris, and S.S.P. Parkin, Phys. Rev. Lett. 90, 256603 (2003).

${ }^{22}$ S. Datta and B. Das, Appl. Phys. Lett. 56, 665 (1990).

${ }^{23}$ C. Ciuti, J.P. McGuire, and L.J. Sham, Phys. Rev. Lett. 89, 156601 (2002).

${ }^{24}$ J. Schliemann, J.C. Egues, and D. Loss, Phys. Rev. Lett. 90, 146801 (2003).

${ }^{25}$ W. Shockley, Electrons and Holes in Semiconductros (Van Nostrand, Princeton, 1950).

${ }^{26}$ The concept of a spin-polarized nonmagnetic bipolar junction transistor was envisioned in the theoretical analysis of spin injection across conventional $p-n$ junctions (Ref. 50).

${ }^{27}$ J. Fabian, I. Žutić, and S. Das Sarma, cond-mat/0211639 (unpublished).

${ }^{28}$ J. Fabian, I. Žutić, and S. Das Sarma, cond-mat/0307014 (unpublished).

${ }^{29}$ The current paper is an extended version of Ref. 27.

${ }^{30}$ M.E. Flatte, Z.G. Yu, E. Johnston-Halperin, and D.D. Awschalom, Appl. Phys. Lett. 82, 4740 (2003).

${ }^{31}$ N. Lebedeva and P. Kuivalainen, J. Appl. Phys. 93, 9845 (2003).

${ }^{32}$ J. Gregg et al., J. Magn. Magn. Mater. 175, 1 (1997).

${ }^{33}$ S. Tiwari, Compound Semiconductor Device Physics (Academic Press, San Diego, 1992).

${ }^{34}$ N. Samarth, S.H. Chun, K.C. Ku, S.J. Potashnik, and P. Schiffer, Solid State Commun. 127, 173 (2003).

${ }^{35}$ B. Hershenov, H. von Philipsborn, and H.L. Pinch, IEEE Trans. Magn. 4, 702 (1968).

${ }^{36}$ We thank M. Field for bringing this reference to our attention.

${ }^{37}$ Y. Ohno, I. Arata, F. Matsukura, K. Ohtani, S. Wang, and H. Ohno, Appl. Surf. Sci. 159-160, 308 (2000).

${ }^{38}$ M. Kohda, Y. Ohno, K. Takamura, F. Matsukura, and H. Ohno,
Jpn. J. Appl. Phys., Part 2 40, L1274 (2001).

${ }^{39}$ E. Johnston-Halperin, D. Lofgreen, R.K. Kawakami, D.K. Young, L. Coldren, A.C. Gossard, and D.D. Awschalom, Phys. Rev. B 65, 041306 (2002).

${ }^{40}$ P. Van Dorpe, Z. Liu, W. V. Roy, V. F. Motsnyi, M. Sawicki, G. Borghs, and J. De Boeck (unpublished).

${ }^{41}$ F. Tsui, L. Ma, and L. He, Appl. Phys. Lett. 83, 954 (2003).

${ }^{42}$ B.D. McCombe and R.J. Wagner, Phys. Rev. B 4, 1285 (1971).

${ }^{43}$ H. Saito, V. Zayets, S. Yamagata, and K. Ando, Phys. Rev. Lett. 90, 207202 (2003).

${ }^{44}$ Early prototypes of $\mathrm{GaAs} /(\mathrm{Ga}, \mathrm{Mn})$ As-based MBT's have recently been fabricated. M. Field (private communication).

${ }^{45}$ J. Fabian, I. Žutić, and S. Das Sarma, Phys. Rev. B 66, 165301 (2002).

${ }^{46}$ R.H. Silsbee, Bull. Magn. Reson. 2, 284 (1980).

${ }^{47}$ M. Johnson and R.H. Silsbee, Phys. Rev. Lett. 55, 1790 (1985).

${ }^{48}$ Optical Orientation, edited by F. Meier and B.P. Zakharchenya (North-Holland, New York, 1984).

${ }^{49}$ D.J. Hilton and C.L. Tang, Phys. Rev. Lett. 89, 146601 (2002).

${ }^{50}$ I. Žutić, J. Fabian, and S. Das Sarma, Phys. Rev. B 64, 121201 (2001).

${ }^{51}$ I. Žutić, J. Fabian, and S. Das Sarma, Phys. Rev. Lett. 88, 066603 (2002).

${ }^{52}$ I. Žutić, J. Fabian, and S. Das Sarma, Appl. Phys. Lett. 79, 1558 (2001).

${ }^{53}$ Spin Dependent Transport in Magnetic Nanostructures, edited by S. Maekawa and T. Shinjo (Taylor and Francis, New York, 2002).

${ }^{54}$ I. Žutić and J. Fabian, Mater. Trans., JIM 44, 2062 (2003).

${ }^{55}$ I. Žutić, J. Fabian, and S. Das Sarma, Appl. Phys. Lett. 82, 221 (2003).

${ }^{56}$ E.I. Rashba, Phys. Rev. B 62, R16 267 (2000).

${ }^{57}$ E.I. Rashba, Eur. Phys. J. B 29, 513 (2002).

${ }^{58}$ Y.V. Pershin and V. Privman, Phys. Rev. Lett. 90, 256603 (2003). 